\title{
A BOX SPLINE CALCULUS FOR COMPUTED TOMOGRAPHY
}

\author{
Alireza Entezari ${ }^{1}$, Michael Unser ${ }^{2}$ \\ 1: CISE Department, University of Florida, Gainesville, FL 32611-6120, USA \\ 2: Biomedical Imaging Group, EPFL, CH-1015, Lausanne, Switzerland
}

\begin{abstract}
B-splines are attractive basis functions for the continuousdomain representation of biomedical images and volumes. In this paper, we prove that the extended family of box splines are closed under the Radon transform and derive explicit formulae for their transforms. Our results are general; they cover all known brands of compactly-supported box splines (tensorproduct B-splines, separable or not) in any dimensions. In particular, we prove that the 2-D Radon transform of an $N$ direction box spline is generally a (non-uniform) polynomial spline of degree $N-1$. The proposed framework allows for a proper discretization of a variety of 2-D and 3-D tomographic reconstruction problems in a box spline basis. It is of relevance for imaging modalities such as X-ray computed tomography and 3-D cryo-electron microscopy.
\end{abstract}

Index Terms - Radon transform, tomography, box splines

\section{INTRODUCTION}

Biomedical imaging heavily relies on tomographic algorithms for the reconstruction of 2-D and 3-D images from projection data [1]. Prominent medical imaging examples are Xray computed tomography, emission tomography (PET and SPECT), and portal imaging for radio therapy. Tomographic reconstruction is also relevant for biology; for instance, for small animal imaging using micro scanners and molecular structure determination from 3-D cryo-electon tomography.

When the projection angles are evenly distributed, the tomogram is usually reconstructed by filtered backprojection (FBP) [1]. When the acquisition conditions are less ideal (e.g., noisy and/or missing data, non-even angular distribution of the projections), it is better to apply iterative techniques such as ART (algebraic reconstruction) or the statistical OSEM method, which offer greater flexibility.

A crucial step in the design of iterative reconstruction algorithms is the discretization of the forward model. This is usually achieved by selecting image-domain basis functions and by mathematically simulating the acquisition pro-

This work was partially supported by the Swiss National Science Foundation under Grant 200020-109415, the Center for Biomedical Imaging (CIBM) and the foundations Leenaards and Louis-Jeantet. cess on them. For a parallel projection geometry, the suitable model is the Radon transform (or the X-ray transform in 3D). Current reconstruction algorithms typically use the natural (square) pixel basis or some blob (or Kaiser-Bessel) functions which have the advantage of being circularly symmetric. While these particular choices simplify the derivation of the forward model, they are not as favorable from an approximation theoretic point of view. Both bases have - at best-a first order of approximation which implies that the rate of decay of the discretization error as the reconstruction grid gets finer is relatively slow $[2,3]$. In principle, choosing higher-order $\mathrm{B}$-spline basis functions would be more advantageous since these are optimal in the sense of their support being minimal for a given order of approximation [3]. Splines have been found to be useful for improving the performance of FBP reconstruction $[4,5,6]$, but have hardly been deployed in the context of iterative algorithms, probably due to the increased complexity of the corresponding forward model.

The contribution of this work is to provide a general characterization of the Radon (or X-ray) transform for the extended family of box splines. While bringing in this level of generality may look like overkill at first sight, we find that it actually simplifies the analysis because the family happens to be closed under the Radon/X-ray transform. Since all commonly-used brands of B-splines are special instances of box splines [7], it therefore makes sense to investigate these functions in detail to obtain a complete analytical picture.

\section{PROJECTION GEOMETRY}

The X-ray transform relates a function to its projections along some directions $\boldsymbol{\theta}$. The projections (or shadows) are obtained by integrating the function along a set of parallel rays. In 2-D, this is equivalent to performing the Radon transform.

To specify the geometry in the general $d$-dimensional setting, we introduce the unit vector $\boldsymbol{\theta} \in \mathbb{R}^{d}$ that points along the direction of integration. The spatial coordinates of the input function are denoted by $\boldsymbol{x}=\left(x_{1}, x_{2}, \ldots, x_{d}\right)$; these are also expressed in a rotated coordinate system as $\boldsymbol{x}=t \boldsymbol{\theta}+\mathbf{P}_{\boldsymbol{\theta}^{\perp}}^{T} \boldsymbol{y}$ where $\mathbf{P}_{\boldsymbol{\theta}^{\perp}}^{T}$ is a $d \times(d-1)$ matrix specifying an orthogonal basis of the hyperplane perpendicular to $\boldsymbol{\theta}$. The corresponding hyperplane coordinates are $\boldsymbol{y}=\left(y_{1}, \ldots, y_{d-1}\right)=\mathbf{P}_{\boldsymbol{\theta}^{\perp}} \boldsymbol{x}$; they are obtained by projecting $\boldsymbol{x}$ onto the basis vectors per- 
pendicular to $\theta$.

The ( $d$-dimensional) $\mathrm{X}$-ray transform $\mathcal{P}$ maps a function $f(\boldsymbol{x}), \boldsymbol{x} \in \mathbb{R}^{d}$ into the set of its line integrals [1]. Specifically, if $\boldsymbol{\theta} \in S^{d-1}$ (e.g., $\boldsymbol{\theta} \in \mathbb{R}^{d}$ with $\|\boldsymbol{\theta}\|=1$ ), then

$$
\mathcal{P}\{f\}(\boldsymbol{y} ; \boldsymbol{\theta})=\int_{\mathbb{R}} f\left(t \boldsymbol{\theta}+\mathbf{P}_{\boldsymbol{\theta}^{\perp}}^{T} \boldsymbol{y}\right) \mathrm{d} t,
$$

with $\boldsymbol{y} \in \mathbb{R}^{d-1}$. In the sequel, we will also use the short-hand notation $\mathcal{P}_{\boldsymbol{\theta}} f(\boldsymbol{y})$ for the above map. The projection geometry for the 2-D case is illustrated in Figure 1; the direction of integration is $\theta=(-\sin \theta, \cos \theta)$ and the projection matrix onto the Radon coordinate system is $\mathbf{P}_{\boldsymbol{\theta}^{\perp}}=\left[\begin{array}{ll}\cos \theta & \sin \theta\end{array}\right]$ so that $y=x_{1} \cos \theta+x_{2} \sin \theta$.

The X-ray transform is a bounded linear operator that has the convenient property of pseudo-commuting with the convolution and translation operations:

$$
\begin{gathered}
\mathcal{P}_{\boldsymbol{\theta}}\{f * g\}(\boldsymbol{y})=\left(\mathcal{P}_{\boldsymbol{\theta}} f * \mathcal{P}_{\boldsymbol{\theta}} g\right)(\boldsymbol{y}), \\
\mathcal{P}_{\boldsymbol{\theta}}\left\{f\left(\cdot-\boldsymbol{x}_{0}\right)\right\}(\boldsymbol{y})=\mathcal{P}_{\boldsymbol{\theta}}\{f\}\left(\boldsymbol{y}-\mathbf{P}_{\boldsymbol{\theta}^{\perp}} \boldsymbol{x}_{0}\right) .
\end{gathered}
$$

These properties are essential to the present work where we are aiming at obtaining a closed-form expression for the Xray transform of a function that is expressed in an integer-shift invariant basis

$$
f(\boldsymbol{x})=\sum_{\boldsymbol{k} \in \mathbb{Z}^{d}} c_{\boldsymbol{k}} \varphi(\boldsymbol{x}-\boldsymbol{k})
$$

where the generator $\varphi$ is a tensor-product B-spline or, more generally, a box spline. The direct application of (3) yields

$$
\mathcal{P}_{\boldsymbol{\theta}} f(\boldsymbol{y})=\sum_{\boldsymbol{k} \in \mathbb{Z}^{d}} c_{\boldsymbol{k}} \mathcal{P}_{\boldsymbol{\theta}}\{\varphi\}\left(\boldsymbol{y}-\mathbf{P}_{\boldsymbol{\theta}^{\perp}} \boldsymbol{k}\right) .
$$

This means that we can discretize the X-ray reconstruction problem exactly, provided that we have an explicit formula for $\mathcal{P}_{\boldsymbol{\theta}}\{\varphi\}(\boldsymbol{y})$, the X-ray (or Radon) transform of the B-spline generator. It is then possible to use (5) to form the system matrix $\mathbf{H}$ that relates the line integrals $p_{m, n}=\mathcal{P}_{\boldsymbol{\theta}_{m}} f\left(\boldsymbol{y}_{n}\right)$ to the $\mathrm{B}$-spline coefficients of the signal $c_{\boldsymbol{k}}$. Note that the approach is also applicable to nonparallel geometries (e.g., fan beam or cone beam), as long as the measurements correspond to pure line integrals.

\section{BOX SPLINES REVIEW}

Geometrically, a box spline is the shadow (i.e., X-ray image) of a hypercube, in $\mathbb{R}^{N}$, when projected to a lowerdimensional space, $\mathbb{R}^{d}(N \geq d)$. A box spline is defined for a set of $N$ vectors $\boldsymbol{\xi}_{1}, \boldsymbol{\xi}_{2}, \ldots, \boldsymbol{\xi}_{N}$ in $\mathbb{R}^{d}$. Each of these vectors is the shadow of an edge of the $N$-hypercube adjacent to its

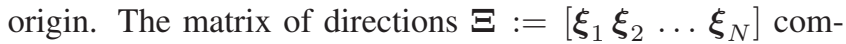
pletely specifies the box spline in $\mathbb{R}^{d}$. Note that the vectors in

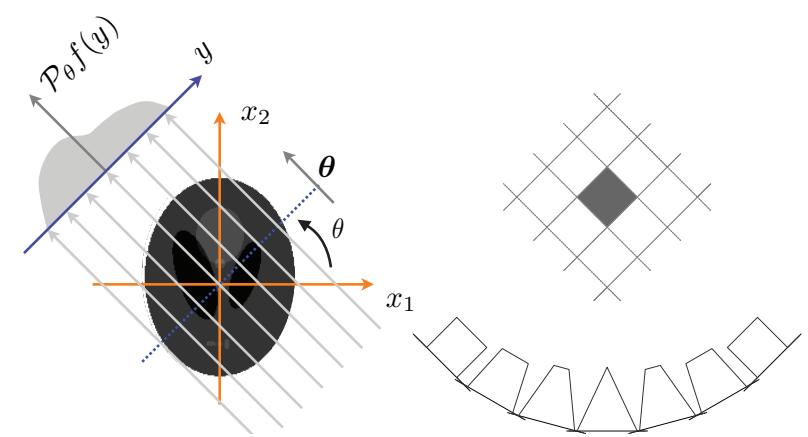

Fig. 1. X-ray projection geometry for $d=2$. On the right: projection of a pixel (tensor-product first-order B-spline) on various angles.

this (multi-) set need not be distinct as they can appear with some multiplicity. When $N=d$, the box spline is simply the (normalized) indicator function of the parallelepiped formed by $d$ vectors in $\mathbb{R}^{d}$ :

$M_{\Xi}(\boldsymbol{x})=\left\{\begin{array}{ll}\frac{1}{|\operatorname{det} \boldsymbol{\Xi}|} & \boldsymbol{x}=\sum_{n=1}^{d} t_{n} \boldsymbol{\xi}_{n} \text { for some } 0 \leq t_{n} \leq 1 . \\ 0 & \text { otherwise }\end{array}\right.$.

For $N>d$, box splines are defined recursively by a "directional" convolution which makes them particularly suitable for the Radon transform:

$$
M_{\Xi \cup \boldsymbol{\xi}}(\boldsymbol{x})=\int_{0}^{1} M_{\Xi}(\boldsymbol{x}-t \boldsymbol{\xi}) \mathrm{d} t .
$$

When the lower dimensional space is $\mathbb{R}$ (i.e., $d=1$ ), the box splines coincide with univariate B-splines (basic splines). When the distinct column vectors of $\boldsymbol{\Xi}$ are orthogonal to each other, box splines amount to tensor-product B-splines. The continuity and approximation power of the spline space formed by the linear combination of shifts of a box spline are determined based on properties of the matrix $\Xi$. The approximation properties, along with the de Boor-Höllig's recurrence relations for fast evaluation of box splines, are discussed in [7] which is the definitive reference on the subject. There are several algorithms for efficient and stable evaluation of box splines $[8,9,10]$.

Another way of constructing box splines, which is probably more transparent to engineers, is by repeated convolution of elementary line-segment-like distributions. Specifically, we have

$$
M_{\Xi}(\boldsymbol{x})=\left(M_{\boldsymbol{\xi}_{1}} * \cdots * M_{\boldsymbol{\xi}_{N}}\right)(\boldsymbol{x})
$$

where the elementary box splines, $M_{\boldsymbol{\xi}_{n}}$, are Dirac-like line distributions supported over $\boldsymbol{x}=t \boldsymbol{\xi}_{n}$ with $t \in[0,1]$. These elementary box splines are in direct geometric correspondence (via a rotation and a proper scaling) with the primary box spline

$$
M_{\vec{e}_{1}}(\boldsymbol{x})=\operatorname{box}\left(x_{1}\right) \delta\left(x_{2}, \cdots, x_{d}\right)
$$


where $\delta\left(x_{2}, \cdots, x_{d}\right)$ is the $(d-1)$-dimensional Dirac distribution and

$$
\operatorname{box}(x)=\left\{\begin{array}{ll}
1 & 0 \leq x \leq 1 \\
0 & \text { otherwise }
\end{array} .\right.
$$

Moreover, they integrate to 1 which is a property that is shared by all box splines (and also preserved through convolution).

Based on (7), one directly infers that the box splines are positive, compactly-supported functions. Their support is a zonotope, which is the Minkowski sum of $N$ vectors in $\boldsymbol{\Xi}$. The center of the support of $M_{\boldsymbol{\Xi}}(\boldsymbol{x})$ is given by $\boldsymbol{x}_{\boldsymbol{\Xi}, 0}:=$ $\frac{1}{2} \sum_{n=1}^{N} \boldsymbol{\xi}_{n}$. The Fourier transform of the box spline is therefore given by:

$$
\hat{M}_{\Xi}(\boldsymbol{\omega})=e^{-j\left\langle\boldsymbol{\omega}, x_{\Xi, 0}\right\rangle} \prod_{n=1}^{N} \operatorname{sinc}\left(\frac{\left\langle\boldsymbol{\omega}, \boldsymbol{\xi}_{n}\right\rangle}{2 \pi}\right),
$$

where $\omega:=\left(\omega_{1}, \ldots, \omega_{d}\right)$ is the multivariate frequency vector.

\section{X-RAY PROJECTION OF BOX SPLINES}

We now turn to our main objective, which is the derivation of an explicit formula for $\mathcal{P}_{\boldsymbol{\theta}}\left\{M_{\boldsymbol{\Xi}}\right\}(\boldsymbol{y})$ where $M_{\boldsymbol{\Xi}}(\boldsymbol{x})$ is a given box spline generator specified by $N$ direction vectors $\boldsymbol{\xi}_{n} \in \boldsymbol{\Xi}$. In the following discussion, $\boldsymbol{\omega}^{\prime}=\left(\omega_{1}^{\prime}, \ldots, \omega_{d-1}^{\prime}\right)$ denotes the $(d-1)$-variate frequency vector corresponding to the projection-domain spatial coordinate vector $\boldsymbol{y} \in \mathbb{R}^{d-1}$, while the projection geometry is the specified in Section 2.

Theorem 1. The X-ray transform of a $d$-variate box spline specified by the direction set, $\Xi$, is a $(d-1)$-variate box spline whose direction set, $\boldsymbol{\Xi}^{\prime}$, is the geometric projection of the former. Specifically,

$$
\mathcal{P}_{\boldsymbol{\theta}}\left\{M_{\boldsymbol{\Xi}}\right\}(\boldsymbol{y})=M_{\mathbf{P}_{\boldsymbol{\theta}} \boldsymbol{\Xi}}(\boldsymbol{y}) .
$$

where $\mathbf{P}_{\boldsymbol{\theta}^{\perp}}$ is the transformation matrix that geometrically projects the $\boldsymbol{x}$-coordinate system onto the $\boldsymbol{y}$-coordinate system perpendicular to $\boldsymbol{\theta}$.

Proof. We start with the derivation of the X-ray transform of the elementary (Dirac-type) box spline $M_{\boldsymbol{\xi}}(\boldsymbol{x})$ whose distributional Fourier transform is

$$
\hat{M}_{\boldsymbol{\xi}}(\boldsymbol{\omega})=e^{-j \frac{\langle\boldsymbol{\omega}, \boldsymbol{\xi}\rangle}{2}} \operatorname{sinc}\left(\frac{\langle\boldsymbol{\omega}, \boldsymbol{\xi}\rangle}{2 \pi}\right) .
$$

We can proceed geometrically by determining the "shadow" of the direction vector $\boldsymbol{\xi}$ since the latter specifies the support of the elementary box spline as a line segment in $\mathbb{R}^{d}$. The alternative is to apply the central slice theorem which states that the Fourier transform of $\mathcal{P}_{\boldsymbol{\theta}} \varphi(\boldsymbol{y})$ corresponds to the restriction of $\hat{\varphi}(\boldsymbol{\omega})$ to the hyperplane perpendicular to $\boldsymbol{\theta}$. Specifically, we have that

$$
\widehat{\mathcal{P}_{\boldsymbol{\theta}} M_{\boldsymbol{\xi}}}\left(\boldsymbol{\omega}^{\prime}\right)=\left.\hat{M}_{\boldsymbol{\xi}}(\boldsymbol{\omega})\right|_{\boldsymbol{\omega}=\mathbf{P}_{\boldsymbol{\theta}}^{T} \boldsymbol{\omega}^{\prime}}=\hat{M}_{\mathbf{P}_{\boldsymbol{\theta}^{\perp}} \boldsymbol{\xi}}\left(\boldsymbol{\omega}^{\prime}\right) .
$$

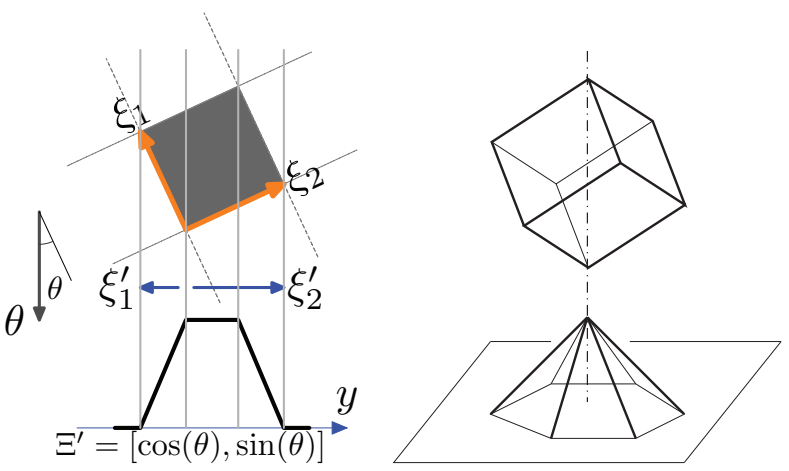

Fig. 2. The X-ray transform of a box spline is a box spline whose directions are projections of the directions of the original box spline onto the projected plane. On the right: a trivariate box spline (a tensor-product B-spline) projected to 2-D.

Since $\left\langle\mathbf{P}_{\boldsymbol{\theta}^{\perp}}^{T} \boldsymbol{\omega}^{\prime}, \boldsymbol{\xi}\right\rangle=\left\langle\boldsymbol{\omega}^{\prime}, \mathbf{P}_{\boldsymbol{\theta}^{\perp}} \boldsymbol{\xi}\right\rangle$, we immediately deduce that $\mathcal{P}_{\boldsymbol{\theta}}\left\{M_{\boldsymbol{\xi}}\right\}(\boldsymbol{y})=M_{\mathbf{P}_{\boldsymbol{\theta}} \boldsymbol{\xi}}(\boldsymbol{y})$, which proves the theorem for $N=1$. By noting that $\boldsymbol{\Xi}^{\prime}=\left[\mathbf{P}_{\boldsymbol{\theta}^{\perp}} \boldsymbol{\xi}_{1} \cdots \mathbf{P}_{\boldsymbol{\theta}^{\perp}} \boldsymbol{\xi}_{N}\right]=$ $\mathbf{P}_{\boldsymbol{\theta}^{\perp}} \boldsymbol{\Xi}$, we are then able to transfer the result to the general case using convolution properties (2) and (7).

The theorem is illustrated in Figure 2. The box spline on the right is a trivariate tensor-product B-spline (first order) whose direction vectors are $(1,0,0),(0,1,0)$ and $(0,0,1)$. When projected to the plane orthogonal to $\boldsymbol{\theta}$, it yields a bivariate, three-direction, box spline that is a hat function with hexagonal support. Likewise, the X-ray transform of the trilinear B-spline (second order) is again a three-direction box spline, but with multiplicity of 2 . The concept carries over to higher-order tensor-product B-splines which are transformed into three-direction box splines with repeated directions, the main point being that these can be evaluated efficiently.

\section{EXPLICIT FORMULAE IN 2-D}

For $d=2$, we will now show that the X-ray transforms of box splines are polynomial splines of degree $N-1$. The geometric configuration is the one shown in Figure 1 with the $\boldsymbol{x}$-to- $y$ projection matrix given by $\mathbf{P}_{\boldsymbol{\theta}^{\perp}}=\left[\begin{array}{ll}\cos \theta & \sin \theta\end{array}\right]$. The application of Theorem 1 together with the convolution formula (7) yields

$$
\mathcal{P}_{\boldsymbol{\theta}} M_{\Xi}(y)=\left(M_{\xi_{1}^{\prime}} * M_{\xi_{2}^{\prime}} * \cdots * M_{\xi_{N}^{\prime}}\right)(y)
$$

with $\xi_{n}^{\prime}=\mathbf{P}_{\boldsymbol{\theta}^{\perp}} \boldsymbol{\xi}_{n}=\left[\boldsymbol{\xi}_{n}\right]_{1} \cos \theta+\left[\boldsymbol{\xi}_{n}\right]_{2} \sin \theta$, and

$$
M_{\xi_{n}^{\prime}}(y)=\frac{1}{\left|\xi_{n}^{\prime}\right|} \operatorname{box}\left(\frac{y}{\xi_{n}^{\prime}}\right)
$$

which is a rectangular box of width $\xi_{n}^{\prime}$ when $\xi_{n}^{\prime} \neq 0$. Note that the convolution factors with $\xi_{n}^{\prime}=0$ may be eliminated 
from (8) since $M_{0}(y)=\delta(y)$. To evaluate the above convolution product, we write $M_{\xi_{n}^{\prime}}(y)$ as

$$
M_{\xi_{n}^{\prime}}(y)=\Delta_{\xi_{n}^{\prime}} u(y)
$$

where $\Delta_{h} f(y)=\frac{f(y)-f(y-h)}{|h|}$ is the finite-difference operator with step $h$, and where $u(y)$ is the unit-step (or Heaviside) function. By substituting (9) in (8), we find that

$$
\begin{aligned}
\mathcal{P}_{\boldsymbol{\theta}} M_{\Xi}(y) & =\left(\Delta_{\xi_{1}^{\prime}} u * \cdots * \Delta_{\xi_{N}^{\prime}} u\right)(y) \\
& =\frac{\Delta_{\xi_{1}^{\prime}} \cdots \Delta_{\xi_{N}^{\prime}} y_{+}^{N-1}}{(N-1) !}
\end{aligned}
$$

where we have used the fact that the $(n+1)$-fold convolution of a step function is $\frac{y_{+}^{n}}{n !}$ with $y_{+}^{n}=\max (y, 0)^{n}$. Finally, we may expand the finite-difference operators which yields a linear expansion of $\mathcal{P}_{\boldsymbol{\theta}} M_{\boldsymbol{\Xi}}(y)$ in terms of some shifted versions of $y_{+}^{N-1}$. The result therefore implies that $\mathcal{P}_{\boldsymbol{\theta}} M_{\boldsymbol{\Xi}}(y)$ is a non-uniform polynomial spline of degree $N-1$, or less if some $\xi_{n}^{\prime}=0$. We can also infer that this box spline function is bell-shaped and that its support is $\sum_{n=1}^{N} \xi_{n}^{\prime}$.

A case of special interest is when the 2-D basis function (or generator) is a tensor-product B-spline of degree $n: \varphi(\boldsymbol{x})=\beta^{n}\left(x_{1}\right) \beta^{n}\left(x_{2}\right)$ [11]. In the present formalism, this corresponds to a box spline with directions vectors $\boldsymbol{\xi}_{1}=(1,0)$ and $\boldsymbol{\xi}_{2}=(1,0)$, each having a multiplicity $(n+1)$ so that $N=2 n+2$. The specialization of (10) for these particular values yields an explicit formula for the Radon transform of a separable B-spline of degree $n$ :

$$
\mathcal{P}_{\theta}\left\{\beta^{n}\left(x_{1}\right) \beta^{n}\left(x_{2}\right)\right\}(y)=\frac{\Delta_{\cos \theta}^{n+1} \Delta_{\sin \theta}^{n+1} y_{+}^{2 n+1}}{(2 n+1) !}
$$

which corresponds to the spline bikernel identified by Horbelt et al. in [5]. The more general result (10), which is valid for any 2-D box spline, including the Zwart-Powell element [6], is new to the best of our knowledge. Similar explicit expansions can be derived for 3-D to 2-D box splines [9, 10].

\section{DISCUSSION}

The choice of the box spline generator in (4) should be dictated by computational and approximation theoretic considerations. Basis functions with larger support and smoothness usually offer better approximation quality, but they also require more computations. This suggests the possibility of a tradeoff between approximation order and the density of the reconstruction grid. In particular, it has been demonstrated that it is computationally advantageous asymptotically to switch to a higher-order basis function than to increase the sampling rate [5].

Tensor-product B-splines constitute a preferred set of basis functions because they are made up from univariate Bsplines building blocks which are widely studied and efficient to evaluate. We should note, however, that the present box spline framework includes other non-separable basis functions with increased isotropy and same approximation order, but lower polynomial degree and smaller support than their tensor-product counterparts. This directly relates to the efficiency issue, which deserves further investigation.

Another concept for consideration is the geometry of the reconstruction lattice. While the common solution is to use a Cartesian grid, the 2-D hexagonal lattice and the 3-D Body Centered Cubic (BCC) and Face Centered Cubic (FCC) lattices have been demonstrated to outperform the sampling efficiency of the Cartesian lattice due to their densest spectral packing properties. The present box spline formalism can perfectly handle those lattices as well. A possible option would be to discretize the tomographic reconstruction problem using the BCC/FCC box splines investigated in [10] since these are quite favorable from the sampling and computational aspects.

\section{References}

[1] F. Natterer, The mathematics of computerized tomography, Society for Industrial and Applied Mathematics, Philadelphia, PA, USA, 2001.

[2] E. Meijering, "Quantitative evaluation of convolutionbased methods for medical image interpolation," Med. Img. Ana., vol. 5, no. 2, pp. 111-126, June 2001.

[3] P. Thévenaz, T. Blu, and M. Unser, "Interpolation revisited," IEEE Transactions on Medical Imaging, vol. 19, no. 7, pp. 739-758, July 2000.

[4] M. Lautsch, "A spline inversion formula for the Radon transform," SIAM J. Numer. Anal., vol. 26, no. 2, pp. 456-467, 1989.

[5] S. Horbelt, M. Liebling, and M. Unser, "Discretization of the radon transform and of its inverse by spline convolutions," IEEE Transactions on Medical Imaging, vol. 21, no. 4, pp. 363-376, 2002.

[6] M. Richter, "Use of box splines in computer tomography," Computing, vol. 61, no. 2, pp. 133-150, 1998.

[7] C. de Boor, K. Höllig, and S. Riemenschneider, Box Splines, vol. 98 of Applied Mathematical Sciences, Springer-Verlag, New York, 1993.

[8] L. Kobbelt, "Stable Evaluation of Box Splines," Numerical Algorithms, vol. 14, no. 4, pp. 377-382, 1997.

[9] L. Condat and D. Van De Ville, "Three-directional box-splines: Characterization and efficient evaluation," IEEE Sig. Proc. Let., vol. 13, no. 7, pp. 417-420, 2006.

[10] A. Entezari, D. Van De Ville, and T. Möller, "Practical box splines for reconstruction on the body centered cubic lattice," IEEE Trans. Visualization and Comp. Graph., vol. 14, no. 2, pp. 313-328, March-April 2008.

[11] M. Unser, A. Aldroubi, and M. Eden, "B-Spline signal processing: Part I-Theory," IEEE Transactions on Signal Processing, vol. 41, no. 2, pp. 821-833, Feb. 1993. 